e0493 TO EXPLORE THE RELATIONSHIP BETWEEN HIGHSENSITIVITY C-REACTIVE PROTEIN AND THE PROGNOSIS OF PATIENTS WITH ACUTE MYOCARDIAL INFARCTION AFTER PERCUTANEOUS CORONARY INTERVENTION

doi:10.1136/hrt.2010.208967.493

Sun Xiao-dan, Zhang Yue-lan. Department of Cardiology Medicine, China Medical University Graduate School

Objective To explore the relationship between high-sensitivity Creactive protein and the prognosis of patients with acute myocardial infarction after percutaneous coronary intervention.

Methods The study comprised 100 consecutive patients with first attack of acute myocardial infarction. They underwent primary PCI within $12 \mathrm{~h}$ after the onset of chest pain. According to their serum hs-CRP level, these patients were divided into three groups: A Group (hs-CRP $<3 \mathrm{mg} / \mathrm{L}, \mathrm{n}=18$ ), B Group (3 mg/l $\leq$ hs-CRP $<10 \mathrm{mg} / \mathrm{l}$, $\mathrm{n}=32$ ) and $C$ Group (hs-CRP $>10 \mathrm{mg} / \mathrm{l}, \mathrm{n}=50$ ). The incidence of MACE within 180 postoperative days was followed.

Results There were no significant differences in age, sex, smoke, hyperlipidaemia, diabetes mellitus, Cardiac troponin I and Lowdensity lipoprotein cholesterol among the three groups $(p>0.05)$. There were significant differences in the serum hs-CRP levels. Left ventricular ejection fraction, hypertension, left anterior descending coronary atery and anterior wall $(\mathrm{p}<0.05)$. Follow-up for 180 days showed that there were significant differences in the incidence of heart failure within 30 days or 180 days and revascularisation rate within 180 days among the three groups $(p<0.05)$. Using Binary logistic regression analysis, by step-back (LR) method, indicated that high concentration of hs-CRP remained an independent predicator of MACE during hospitalisation and within 30 days $(\mathrm{OR}=2.42,95 \%$ $\mathrm{CI}=1.020$ to $5.746, \mathrm{p}=0.045) ;(\mathrm{OR}=2.187,95 \% \mathrm{CI} 1.028$ to 4.653 , $\mathrm{p}=0.042$ ). It is a more useful predictor for the incidence of heart failure within 30 days $(\mathrm{OR}=2.565,95 \% \quad \mathrm{CI}=1.032$ to 6.375 , $\mathrm{p}=0.043$ ).

Conclusion High level hs-CRP measured after the primary PCI is the independent predictive factors of MACE for the patients with first attack of AMI during hospitalisation and within 30 days. It has a stronger predictive value, especially for the incidence of heart failure within 30 days. The patients of high level hs-CRP group is more than the normal hs-CRP group in the occurrence of MACE during hospitalisation and within 30 days and the occurrence of heart failure within 30 days.

\section{e0494 EXTENSIVE DISSECTION TO THE CORONARY SINUS OF VALSALVA DURING PERCUTANEOUS INTERVENTION IN RIGHT CORONARY ARTERY-A CASE REPORT AND LITERATURE REVIEW}

doi:10.1136/hrt.2010.208967.494

${ }^{1} \mathrm{Li} \mathrm{Li},{ }^{2} \mathrm{Cao}$ Yunshan. ${ }^{1}$ People's Hospital of Gansu Province, ${ }^{2}$ Peoples Hospital of Gansu Province

Severe retrograde dissection extending into the sinus of Valsalva is a rare complication during percutaneous coronary intervention (PCI), but life threatening. The danger of this event depends on two aspects: first, the potential occlusion of the related coronary artery and second, the possibility of the dissection extending to the ascending aorta and further. There is some literature about this complication, but this particular complication has not been previously reported in China. We think that the rarity and unknown significance of this complication have probably led to a general underreporting in our country. We present a case of coronary artery dissection during a PCI in which progressively extended retrogradely into the sinus of valsalva, and was successfully treated with stenting without an operation.

\section{e0495 TRANSCATHETER CLOSURE OF LARGE PATENT DUCTUS ARTERIOSUS WITH SEVERE PULMONARY ARTERIAL HYPERTENSION IN ADULTS: TWO-YEAR FOLLOW-UP RESULTS}

doi:10.1136/hrt.2010.208967.495

${ }^{1}$ Zhang Caojin, ${ }^{2}$ Huang Yigao, ${ }^{2}$ Huang Xinsheng, ${ }^{2}$ Huang Tao, ${ }^{2}$ Shen Junjun, ${ }^{2}$ Huang Wenhui. ${ }^{1}$ Guangdong General Hospital, Guangdong Cardiovascular Institute; ${ }^{2}$ Guangdong General Hospital Guangdong Cardiovascular Institute

Aim To evaluate the immediate and follow-up results of transcatheter closure of large PDAs with severe pulmonary arterial hypertension (PAH) in adults.

Method After a complete haemodynamic evaluation differentiating from the reversibility of severe PAH, transcatheter closure of PDA was performed. Patients were followed up clinically and echocardiographically at 24 h, 1 month, 3 months, 6 months, 12 months and 24 months after occlusion.

Results 38 patients had successful occlusion, PAP, LVEF and FS significantly decreased immediately after occlusion $(92.5 \pm 23.1 \mathrm{~mm} \mathrm{Hg}$ vs $47.2 \pm 15.7 \mathrm{~mm} \mathrm{Hg}, \mathrm{p}<0.01 ; 66.5 \pm 9.0$ vs $52.2 \pm 10.73, \mathrm{p}<0.05$ and $6.3 \pm 8.8$ vs $28.9 \pm 9.1, p<0.05$, respectively). At 1 month after PDA closure, the signs and symptoms had improved markedly in all 38 patients, and PDAs were completely closed and remained closed during the follow up. 25 patients having different degrees of dyspnoea were treated with ACEI and/or digoxin after occlusion. After 1 to 3 months of peroral drug therapy, their exercise tolerance had improved from NYHA class III-IV to NYHA class I. During follow up, no latent arrhythmias were found, the LAD, LVEDD, LVESD, LVMI and PASP decreased significantly $(p<0.05)$, and FS and LVEF recovered compared to the immediate postclosure state. However, FS and LVEF remained low compared to the preclosure state.

Conclusion Transcatheter closure of large PDA with severe PAH is feasible, effective, and safe in the adults. Significant LV systolic changes may occur after closure of large PDA, and LV function usually recovers within a few months. Further study should be performed.

\section{e0496 COMPARATIVE STUDY OF IMPAIRED WITH NORMAL LEFT VENTRICLE FUNCTION PATIENTS WITH TRIPLE CORONARY ARTERY DISEASE}

doi:10.1136/hrt.2010.208967.496

Gao Zhan, Yang Yue-Jin, Xu Bo, Chen Ji-Lin, Qiao Shu-Bin, Wu Yong-Jian, Qin Xuewen, Yao Min, Liu Hai-bo, Yuan Jin-Qing, Chen Jue, You Shi-Jie, Dai Jun, Li Jian-Jun, Gao Run-Lin. Department of Cardiology, Cardiovascular Institute \& Fuwai Hospital, Chinese Academy of Medical Sciences, Peking Union Medical College, Beijing, China

Background Ischaemic cardiomyopathy is one of the fatal courses of coronary heart disease, its clinical characteristics and percutaneous coronary intervention (PCI) effect on it still need to be identified.

Methods From April 2004 to April 2007, 4494 consecutive patients with triple coronary arteries disease identified by coronary angiogram $(>70 \%$ stenosis of each vessel) in our center were divided into 
two groups according to left ventricle ejection fraction (LVEF) (N group: $n=4129, \mathrm{LVEF}>40 \%$; L group: $n=365$, LVEF $\leq 40 \%$ ).

Results Patients in L group was younger $(60.6 \pm 9.72$ vs $65.2 \pm 10.7$ years; $p<0.001)$. There was more previous myocardial infarction (MI) and diabetes and less hypertension and hyperlipidaemia in L group. Logistic regression analysis indicated that the age, previous MI, diabetes, previous PCI and hyperlipidaemia were independent indexes to left ventricle function of triple vessel disease. Eighty-three and 2301 patients in $\mathrm{L}$ and $\mathrm{N}$ group $(22.7 \%$ and $55.7 \%$, respectively) were treated with PCI. The follow-up period of $\mathrm{L}$ and $\mathrm{N}$ groups were $581 \pm 298$ and $639 \pm 293$ days, respectively. MACE rate was significantly high in L group ( $38.6 \%$ vs $18.9 \%$; $<<0.001$ ), which was contributed by cardiac death, no fatal MI and TVR $(9.6 \%$ vs $0.9 \% ; p<0.001,7.2 \%$ vs $2.0 \% ; p<0.001$ and $21.7 \%$ vs $16.0 \%$; $\mathrm{p}=0.173$, respectively). There was no difference of total stent thrombosis or its components in both groups (total: $3.9 \%$ vs $3.5 \%$; $p=1.000$, early: $0.2 \%$ vs $0.9 \% ; p=0.256$, late: $0.7 \%$ vs $1.3 \% ; p=0.404$ and very late: $3.1 \%$ vs $1.3 \% ; p=0.201$, respectively). Seven month Angiographic follow-up indicated that both in-stent and in-segment restenosis rate were significantly higher in L group $(21.0 \%$ vs $11.1 \%$; $\mathrm{p}=0.034$ and $24.0 \%$ vs $12.2 \% ; \mathrm{p}=0.018$ ).

Conclusion This one center, large sample study showed clinical characteristics of ischaemic cardiomyopathy, MI and diabetes might contribute to its morbidity, and PCI might prevent its morbidity. PCI of patients with triple coronary arteries disease and impaired left ventricle (LV) function leaded to worse outcomes when compared with normal LV function.

\section{e0497 CLINICAL EVALUATION ON THE SAFETY AND THERAPEUTIC} EFFICACY OF EXCEL DRUG-ELUTING STENT

\section{doi:10.1136/hrt.2010.208967.497}

Jun Tang, Xiaogian Li, Hegui Wang, Xuejun Hu, Wenjun Gao, Jun Wang, Dabin Pan, Yongsheng Ke. Department of Cardiovascular Diseases, Yijishan Hospital, Wannan Medical College, Wuhu

Objective To investigate the clinical safety and mid-and short-term efficacy of rapamycin eluting stent (Excel) in patients with coronary artery disease.

Methods Between Jul. 2006 and Jun. 2009, 240 patients of coronary heart disease received percutaneous coronary intervention (PCI) with Excel stent and were followed up from 6 to 24 months for observing the incidence of angina pectoris, myocardial infarction, sudden death and revascularization.

Results 327 pieces of Excel stent were implanted in 272 target lesions (269 with de novo and 3 of restenosis), but 2 cases failed due to seriously deformed middle anterior descending artery from calcification in 1 and the angle of middle circumflex branch larger than 90 degrees in another to prevent the passage. 325 pieces of stent were successfully implanted, and the postoperative follow-up in the $8^{\text {th }}$ to $18^{\text {th }}$ month showed that angina pectoris occurred in 5 , restenosis in 2 and normal in 3 by coronary arteriongraphy, suspected thrombosis in 1 at the $5^{\text {th }}$ month after the operation regarding ventricular fibrillation, haemorrhage of upper digestive tract in 4 at the $6^{\text {th }}$ month of the intervention, in which 1 underwent inpatient therapy with blood transfusion. The postoperative major coronary adverse event accounted for $4.58 \%$ between 6 and 24 months.

Conclusion Excel drug-eluting stent may be excellent in treatment of coronary artery diseases with regard to its safety and mid-and shortterm effect.

\section{e0498 CASE REPORT: PERCUTANEOUS MULTIPLE OVERLAPPED OVERLENGTH SELF-EXPANDING STENT PLACEMENT TREATMENT SUPERFICIAL FEMORAL ARTERY CHRONIC SUBTOTAL OCCLUSIONS}

doi:10.1136/hrt.2010.208967.498

${ }^{1}$ Jing Tao, ${ }^{2}$ Liu Jian-ping, ${ }^{2}$ Li Yong-hua, ${ }^{2}$ He Guo-xiang, ${ }^{2}$ Song Zhi-yuan, ${ }^{2}$ Chi Lu-xiang. ${ }^{1}$ The Department of Cardiology Southweast Hospital Third Military Medical University \& Chongqing Institute of Interventional Cardiology; ${ }^{2}$ The Department of Cardiology Southweast Hospital Third Military Medical University Chongqing Institute of Interventional Cardiology

PAD is a significant cause of both morbidity and death. In spite of TASC II recommendation that surgery is the preferred treatment for femoro-popliteal Type C\&D lesions, promising clinical results of percutaneous revascularization had been obtained with a low risk of morbidity and mortality. We present a case of multiple overlapped, overlength self-expanding stent (extend as long as $450 \mathrm{~mm}$ ) placement to treat superficial femoral artery (SFA) chronic subtotal occlusions. No stent fractures or restenoses were observed at 1-year follow-up and with a favourable clinical results. Clinical and research medicine.

\section{E0499 LONG TERM CLINICAL FOLLOW-UP OF PATIENTS WITH VERSUS WITHOUT OVERLAPPING DRUG ELUTING STENTS FOR LONG CORONARY LESIONS}

doi:10.1136/hrt.2010.208967.499

Gao Fei, Zhou Yu Jie, Wang Zhi Jian, Liu Xiao Li, Nie Bin, Yang Shi Wei, Jia De An, Yan Zhen Xian. Beijing An Zhen Hospital

Background Drug-eluting stents (DSE) markedly reduce the rate of in stent restenosis, but limited data exists as to the long-term outcome in patients with long coronary lesions $(\geq 40 \mathrm{~mm}$ ) undergoing multiple overlapping DES implantation of in native coronary vessels. Methods A total of 11073 consecutive patients undergoing percutaneous coronary intervention (PCI) with drug eluting stent were retrospectively were screened. Interventions for in stent restenosis, bypass graft, and primary PCI were excluded. All other patients $(n=1411)$ receiving $\geq 2$ overlapping drug-eluting stents and total stent length in target lesion $\geq 40 \mathrm{~mm}$ for de novo coronary arteries were retrospectively analysed. The clinical outcomes of those patients were compared with consecutive patients with multiple drug-eluting stents implantation but without overlapping stents. An age-matched ( \pm 5 years) control group with the same total number of stents and similar total stent length $( \pm 10 \mathrm{~mm})$ in target vessel was collected from the total population undergoing PCI with DES during the same study period. Patients were followed up either by telephone or by visiting outpatient clinic.

Results Successfully matching and complete clinical follow-up was achieved in 522 pairs. Median clinical follow-up duration was 35.0 months (IOR 24.3-46.4); Mean total length and number of stents in target vessel was $65.2 \pm 24.4 \mathrm{~mm}$ and $2.4 \pm 0.7$, respectively. Nineteen percent lesions in the overall cohort were chronic total occlusions and 9\% involved left main or bifurcation lesions. Patients with overlapping DSE stints were associated with significantly increased overall major adverse cardiac event rate (MACE) $(18.4 \%$ vs $11.9 \%, \mathrm{p}=0.004)$, which was largely driven by significantly higher incidence of target vessel revascularisation ( $8.8 \%$ vs $5.2 \%, p=0.028)$. Whereas all cause mortality ( $5.2 \%$ vs $4.2 \%, \mathrm{p}=0.559)$, myocardial infarction $(5.0 \%$ vs $4.0 \%, \mathrm{p}=0.551)$ and ARC defined definite or probable stent thrombosis rates $(2.3 \%$ vs $1.5 \%, \mathrm{p}=0.499)$ were comparable in patients with or without overlapping DES.

Conclusion Coronary stenting for long lesions ( $\geq 40 \mathrm{~mm}$ ) using overlapping drug-eluting stents presented in over $12 \%$ of patients undergoing PCI in routine clinical practice and was associated with acceptable clinical outcome during median 35.0 months follow-up. 